\title{
Feedback control of a motorized skateboard
}

\author{
Pascal Morin, Claude Samson
}

\begin{abstract}
This paper develops a feedback control solution for the automatization of a motorized skateboard, also called symmetric snakeboard in the Robotics and Automatic Control literature. Controlling this system is particularly challenging due to the association of kinematic and dynamic nonintegrable constraints to which it is subjected. The Transverse Function approach, combined with backstepping, is used to derive dynamic feedback laws that ensure practical stabilization of arbitrary reference trajectories in the Cartesian space. Simulation results illustrate the proposed approach.
\end{abstract}

\section{INTRODUCTION}

The difficulties associated with the problem of fixed-point stabilization of mechanical systems subjected to nonintegrable constraints were revealed through the application of a (now famous) Brockett's theorem [1]. Different classes of such systems can be identified. One consists of systems subjected to kinematic constraints only, alike classical wheeled vehicles (unicycle, car, etc). They are often referred to as nonholonomic systems, and controlling them can be brought back to controlling their kinematic model with velocity inputs. This has motivated an abundant literature on feedback control of driftless systems [2]-[10], etc. A second class consists of systems subjected to dynamic constraints only, such as the underactuated spacecraft [11] or the hovercraft [12] (also known as the slider). These systems are often referred to as underactuated systems. Feedback control design must be addressed at the dynamical level, which yields additional difficulties due to the presence of a drift term in the control model. References [13]-[16] account for the relatively small number of papers devoted to the subject. A last class consists of systems subjected to both kinematic and dynamic constraints [17]. Controlling these systems is particularly challenging because it combines the difficulties associated with classical nonholonomic systems and underactuated systems. Most of the literature on this topic was published by the CALTECH group in the mid-nineties [17]-[20], with papers essentially inspired by the study of the so-called snakeboard [18] that remains to this day the archetype of this class of systems. This series of articles laid the foundations of modelling and controllability analyses for these systems. In particular, it is proved in [19] that the snakeboard dynamic model is SmallTime Locally Controllable (STLC) from any equilibrium point. Open-loop control design was also studied in the form of gait generation, i.e., "cyclic pattern of internal shape changes which couple to produce a net motion [19]" (see also [21]).

P. Morin is with Sorbonne Université, CNRS, Institut des Systèmes Intelligents et de Robotique, ISIR, 75005 Paris, France (e-mail: pascal.morinesorbonne-universite.fr).

C. Samson is with INRIA-I3S-CNRS, Université Côte d'Azur, Sophia Antipolis, France (e-mail: claude.samson@inria.fr, csamsonei3s. unice.fr).
Efforts devoted to the design of open-loop control strategies gave rise to a few more papers [22]-[24] involving a richer set of motion primitives, not necessarily reduced to cyclic internal motions. The latter reference, in particular, derives a general analytic solution to the problem of making the system track any (feasible and smooth enough) desired position trajectory.

The literature devoted to the snakeboard points out the attention paid in the past to controllability and open-loop control design issues. By contrast, no feedback control method has been proposed for this system. We are only aware of a stability analysis provided in [25] where the rectilinear motion of a skateboard-skater under the action of a linear PD (Proportional-Derivative) control is studied. The objective of the present paper is to propose a generic solution to the trajectory tracking problem for a motorized skateboard. The method here adopted relies on our previous work on the so-called Transverse Function (TF) approach, which we have already applied to a variety of nonholonomic [10], [26] and underactuated [15] mechanical systems, including snaketype mechanisms like the so-called trident snake robot [27], [28] that bear similarities with the snakeboard in the sense that they rely on the coupling of internal shape variables to produce motion. Illustrative videos are available at the website http://www.transversefunction.com/. However, this approach is for the first time applied to a mechanical system subjected to both kinematic and dynamic nonintegrable constraints. We here consider the case of a skateboard, i.e. a snakeboard for which front and rear wheels are coupled and controlled with opposite angles (also called "symmetric snakeboard" in [24]). By suppressing one degree of freedom this coupling constraint tends to further complicate the control problem. Modeling and open-loop control of the skateboard have also been addressed in several articles [19], [22]. As in previous applications of the TF approach, the dynamic feedback control here proposed allows for the tracking of arbitrary reference pose motions in Cartesian space (i.e., in both position and orientation). Avoiding prior determination of feasible pose trajectories is an important asset because the calculation of such trajectories is not only complex, it is also highly non-robust with respect to modeling errors. The price to be paid is that asymptotic stability (convergence of the tracking errors to zero, in particular) is clearly not achievable when the trajectory is not feasible. The objective of asymptotic stability is accordingly relaxed in favour of a practical stability objective, with the possibility to tune the tracking precision via the control parameters.

The paper is organized as follows. A description of the considered device, and notation used in the paper, are presented in the short preliminary Section II. Modeling of the skateboard is carried out in Section III. The feedback control design approach is developed in Section IV, and illustrating simulation results are presented in Section V. The concluding 


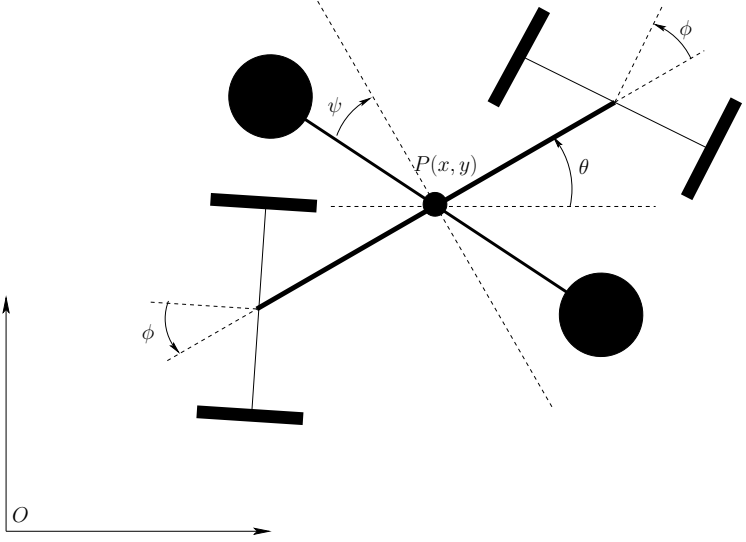

Fig. 1. Skateboard parameters

Section VI is followed by an Appendix detailing the proof of the paper's main result.

\section{DEVICE DESCRIPTION AND NOTATION}

A sketch view from above of the considered motorized skateboard is represented on Fig. 1. The device is essentially composed of a rigid body (the board itself), a set of front and rear driving wheels whose common angle $\phi$ w.r.t. the board's horizontal axis is actuated via the use of one (or two) embarked electric motor(s) in place of the rider's foot movements, and a momentum bar (flywheel or rotor) simulating the movement of a human torso whose vertical axis passes through the center of mass of the device and whose angle $\psi$ w.r.t. the board's axis is also actuated via an embarked electric motor. The position coordinates of the board w.r.t. a fixed frame are $x$ and $y$, and the board's orientation angle is $\theta$. The total mass of the device is $m$, the skateboard body inertia is $J$, and the momentum bar inertia about the vertical axis is $J_{b}$. The half-length of the board is denoted as $l$. The driving wheels masses are assumed to be very small compared to the masses of the other skateboard components and their inertia about the vertical axis is here neglected.

The following notation is used throughout the article.

- $\mathbb{R}^{n}:$ is the $n$-dimensional real vector space, and $x_{i}$, with $i \in\{1, \ldots, n\}$, denotes the $i t h$ component of a vector $x \in \mathbb{R}^{n}$.

- $S O(2):$ is the group of rotations in the $2 \mathrm{D}$ plane.

- $S E(2)$ : is the group of rigid transformations in the $2 \mathrm{D}$ plane, i.e. the semi-direct product $S O(2) \times \mathbb{R}^{2}$. The group product of this 3-dimensional Lie group is defined by:

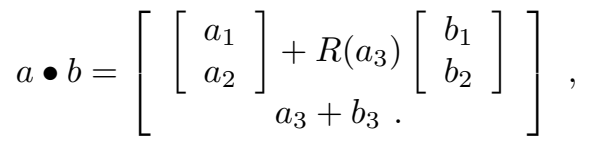

with $\left[a_{1}, a_{2}\right]^{\top}$ and $\left[b_{1}, b_{2}\right]^{\top} \in \mathbb{R}^{2}$, and $a_{3}$ and $b_{3}$ rotation angles. The inverse of an element $g \in S E(2)$ is denoted by $g^{-1}$.

- $\mathbb{T}^{2}$ : denotes the two-dimensional torus;

- $c_{\theta}$ and $s_{\theta}$ stand for $\cos (\theta)$ and $\sin (\theta)$ respectively, to shorten the notation.

\section{MODELING EQUATIONS}

Modeling equations of the skateboard's dynamics based on Lagrange equation have been worked out in several articles, starting with [18]. The first part of this section essentially recalls these equations. A new form of the dynamic equations is subsequently derived to simplify the control design.

The system's kinetic energy of the corresponding simplified skateboard model is thus given by:

$$
\begin{aligned}
E_{c} & =\frac{1}{2} m\left(\dot{x}^{2}+\dot{y}^{2}\right)+\frac{1}{2} J \dot{\theta}^{2}+\frac{1}{2} J_{b}(\dot{\theta}+\dot{\psi})^{2} \\
& =\frac{1}{2} m\left(v_{x}^{2}+v_{y}^{2}\right)+\frac{1}{2} J \dot{\theta}^{2}+\frac{1}{2} J_{b}(\dot{\theta}+\dot{\psi})^{2}
\end{aligned}
$$

with $v_{x}$ and $v_{y}$ the board's velocity coordinates expressed in the mobile frame attached to the board, i.e.

$$
\begin{aligned}
& {\left[\begin{array}{c}
\dot{x} \\
\dot{y} \\
\dot{\theta}
\end{array}\right]=\bar{R}(\theta)\left[\begin{array}{c}
v_{x} \\
v_{y} \\
\dot{\theta}
\end{array}\right]}
\end{aligned}
$$

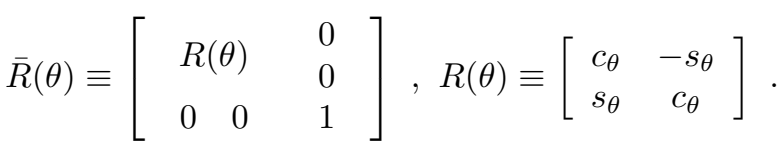

We assume that the driving wheels roll without slipping so that the following nonholonomic kinematic constraints are enforced:

$$
\left[\begin{array}{ccc}
s_{\phi} & 0 & -l c_{\phi} \\
0 & 1 & 0
\end{array}\right]\left[\begin{array}{c}
v_{x} \\
v_{y} \\
\dot{\theta}
\end{array}\right]=0
$$

The dynamic equations of the system are obtained via the Lagrange equation:

$$
\frac{d}{d t}\left(\frac{\partial L}{\partial \dot{q}}\right)=F+A(q) \lambda,
$$

with the Lagrangian $L$ equal to $E_{c}$ in the present case, $\lambda=$ $\left(\lambda_{1}, \lambda_{2}\right)^{\top}$ the vector of Lagrangian parameters associated with the kinematic constraints, $F=\left(0,0,0,0, \tau_{\psi}\right)^{\top}$,

$$
\dot{q}=\left[\begin{array}{c}
v_{x} \\
v_{y} \\
\dot{\theta} \\
\dot{\phi} \\
\dot{\psi}
\end{array}\right], A(q)^{\top}=\left[\begin{array}{ccccc}
s_{\phi} & 0 & -l c_{\phi} & 0 & 0 \\
0 & 1 & 0 & 0 & 0
\end{array}\right],
$$

and $\tau_{\psi}$ the torque applied to the momentum bar. This yields the following set of equations:

$$
\left[\begin{array}{c}
m \dot{v}_{x} \\
m \dot{v}_{y} \\
\left(J+J_{b}\right) \ddot{\theta}+J_{b} \ddot{\psi} \\
J_{b}(\ddot{\theta}+\ddot{\psi})
\end{array}\right]=\left[\begin{array}{c}
s_{\phi} \lambda_{1} \\
\lambda_{2} \\
-l c_{\phi} \lambda_{1} \\
\tau_{\psi}
\end{array}\right] .
$$

It remains to eliminate the Lagrangian parameters from these equations. From $v_{y} \equiv 0$ one already deduces that $\lambda_{2}=0$. From the kinematic constraint $s_{\phi} v_{x}-l c_{\phi} \dot{\theta}=0$ it comes that $\dot{\theta}=\frac{\tan _{\phi}}{l} v_{x}$. Differentiating this latter relation w.r.t. time yields $\ddot{\theta}=\frac{\dot{\phi}}{l c_{\phi}^{2}} v_{x}+\frac{\tan _{\phi}}{l} \dot{v}_{x}$ and, using the first equation of (6), $\ddot{\theta}=$ $\frac{\dot{\phi}}{l c_{\phi}^{2}} v_{x}+\frac{s^{2} \phi}{m l c_{\phi}} \lambda_{1}$. Now, subtracting the fourth equation of (6) from the third one yields $\ddot{\theta}=-\frac{l c_{\phi}}{J} \lambda_{1}-\frac{\tau_{\psi}}{J}$. The elimination 
of $\ddot{\theta}$ between the last two equalities then yields $\lambda_{1}=\frac{m u_{2}}{l c_{\phi}}$ with:

$$
u_{2} \equiv-\frac{1}{m}\left(1+\frac{J}{m}\left(\frac{\tan _{\phi}}{l}\right)^{2}\right)^{-1}\left(\tau_{\psi}+\frac{J \dot{\phi}}{l c_{\phi}^{2}} v_{x}\right)
$$

By denoting the skateboard path curvature as $\eta \equiv \frac{\tan _{\phi}}{l}$ and further defining:

$$
u_{1} \equiv \frac{\dot{\phi}}{l c_{\phi}^{2}}
$$

and:

$$
\bar{\psi} \equiv-\frac{J_{b}}{m}\left(\left(1+\frac{J}{J_{b}}\right) \theta+\psi\right),
$$

we finally obtain the following set of equations characterizing the system's dynamics:

$$
\left\{\begin{array}{l}
\dot{x}_{1}=c_{x_{3}} x_{5} \\
\dot{x}_{2}=s_{x_{3}} x_{5} \\
\dot{x}_{3}=x_{4} x_{5} \\
\dot{x}_{4}=u_{1} \\
\dot{x}_{5}=x_{4} u_{2} \\
\dot{x}_{6}=u_{2} \\
\dot{x}_{7}=x_{6}
\end{array}\right.
$$

with $x_{1} \equiv x, x_{2} \equiv y, x_{3} \equiv \theta, x_{4} \equiv \eta, x_{5} \equiv v_{x}, x_{6} \equiv \dot{\bar{\psi}}$, and $x_{7} \equiv \bar{\psi}$. The variables $u_{1}$ and $u_{2}$ can be interpreted as auxiliary control inputs from which the physical control inputs $\dot{\phi}$ and $\tau_{\psi}$ can be deduced once the driving angle $\phi$ is measured. This writing of the dynamic equations calls for the following remarks.

1) The first four equations in (10) are similar to the kinematic equations of a car-like vehicle. However, contrary to the car, the velocity $v_{x}$ is not directly controlled: it is subjected to the constraint defined by the fifth equation.

2) It follows from (10) that for any smooth enough time functions $x^{*}, y^{*}$, one can define associated time functions $\theta^{*}, v_{x}^{*}, \eta^{*}, \bar{\psi}^{*}, u_{1}^{*}$, and $u_{2}^{*}$ such that $\left(x^{*}, y^{*}, \theta^{*}\right.$, $\left.\eta^{*}, v_{x}^{*}, \bar{\psi}^{*}, \bar{\psi}^{*}\right)$ is a solution to (10) with $u_{1}=u_{1}^{*}$ and $u_{2}=u_{2}^{*}$. The motion planning strategy proposed in [24] is based on this property, although the form (10) of the dynamic model was not identified in this reference. The functions $\theta^{*}, v_{x}^{*}, \eta^{*}, u_{1}^{*}$ and $u_{2}^{*}$ are uniquely defined as:

$$
\begin{gathered}
\theta^{*}=\arctan 2\left(y^{*}, x^{*}\right), v_{x}^{*}=\dot{x}^{*} c_{\theta^{*}}+\dot{y}^{*} s_{\theta^{*}} \\
\eta^{*}=\frac{\dot{\theta}^{*}}{v_{x}^{*}}, u_{1}^{*}=\dot{\eta}^{*}, u_{2}^{*}=\frac{\dot{v}_{x}^{*}}{\eta^{*}} .
\end{gathered}
$$

However, this open-loop control solution presents singularities when either $v_{x}^{*}$ or $\eta^{*}$ crosses zero whose avoidance renders the determination of physically feasible trajectories difficult. Moreover, $\bar{\psi}$ is not uniquely defined: it is only defined up to an affine function of time. This shows that the system is not differentially flat [29]. This issue is not addressed in [24].

Controlling the skateboard then comes to controlling the above system via the control inputs $u_{1}$ and $u_{2}$.

\section{FEEDBACK CONTROL DESIGN}

The primary control objective here addressed is to track a reference trajectory for the skateboard body. More precisely, given a time-parametrized path $\left(x_{r}(t), y_{r}(t), \theta_{r}(t)\right)$ the objective is to have the first three components of the system (10) stabilize this reference path. This should be accomplished by keeping all other state components, the angular velocity of the momentum bar in particular, bounded. A further possible requirement is to keep the angle $\psi$ of the momentum bar also bounded, alike the torso of a human rider. This primary control objective is a standard one. In the last decades it has been addressed in a multitude of publications devoted to the control of various mobile robots (nonholonomic wheeled vehicles of the car-like type, aerial vehicles such as quadrotors and airplanes, ships, submarines,...). However, for the skateboard device here considered, it turns out to be particularly challenging. Why is it so? The first reason is that along the simplest reference trajectories satisfying the system's equations (i.e. along theoretically admissible or feasible trajectories) the linearized error equations associated with this stabilization problem are not controllable. For instance, one easily verifies that such is the case when the reference trajectory consists of moving along a straight line with a constant forward velocity $v_{x}$ and a constant momentum bar angle $\psi$. This already means that classical control methods based on the linearization of the system's equations do not provide a solution in the general case. Even more troublesome is the difficulty to determine feasible trajectories of interest, with the constraint of feasibility being needed to make asymptotic stability a workable control objective via either linear or nonlinear control techniques. This difficulty is related to the fact that the system is not differentially flat. Calculating feasible trajectories becomes even more problematic when one takes into account wheels contact and rolling friction forces that oppose the skateboard's motion. For instance, motion along a straight line with constant forward velocity is then no longer possible (feasible). A third well known difficulty attached to all wheeled vehicles subjected to nonholonomic constraints is that fixed poses (i.e. constant reference positions and orientations of the board) cannot be asymptotically stabilized by means of continuously differentiable pure state feedbacks, by application of a Brockett's theorem [1]. This impossibility can be deduced from the first four equations of the system that coincide with the modeling of a car-like vehicle when $v_{x}$ is taken as a free control input. The aforementioned difficulties may explain why, despite a few illuminating studies devoted to the mathematics underlying the undulatory motion and gait patterns associated with the motorized skateboard (or its snakeboard variant for which front and rear driving wheels angles can be controlled independently) [20], [30], [31], no feedback control scheme has so far been proposed for this type of vehicle. The control design proposed next overcomes these difficulties by abandoning the classical objective of asymptotic stabilization of feasible trajectories and by replacing it by the practical stabilization of any (thus not necessarily feasible) pre-specified reference trajectory of the skateboard body via the combined use of backstepping and so-called transverse functions. 


\section{A. Control design principle}

Prior to going into calculation details it may be useful to expose the general lines of the proposed control design. The approach essentially consists of a two-step backstepping procedure. First the variables $x_{4}$ and $x_{5}$ are taken as control inputs in order to practically stabilize the pose $g \equiv\left[x_{1}, x_{2}, x_{3}\right]^{\top}$ $\left(=[x, y, \theta]^{\top}\right)$ of the skateboard body about the reference pose $g_{r}(t) \equiv\left[x_{r}(t), y_{r}(t), \theta_{r}(t)\right]^{\top}$. This step is equivalent to controlling a car-like vehicle as done in [26]. The resulting control expressions are denoted as $x_{4}^{\star}$ and $x_{5}^{\star}$ respectively. They represent "ideal" control inputs that would be used if the path curvature $\eta$ and the forward velocity $v_{x}$ were both available as unconstrained control inputs. Let $x_{6}^{\star}$ denote a desired velocity for $\dot{\bar{\psi}}$ chosen to yield a desired behaviour for the bar momentum angle when $x_{6}=x_{6}^{\star}$. The second step of the control design consists in determining inputs $u_{1}$ and $u_{2}$ that achieve the practical stabilization of the errors $\tilde{x}_{i} \equiv\left(x_{i}-x_{i}^{\star}\right)$ $(i=4,5,6)$ about zero. This step takes advantage of the fact that the subsystem in the state variables $x_{4}, x_{5}$ and $x_{6}$ is the so-called three-dimensional chained system with two inputs for which feedback controls based on the TF approach have been derived in [26] and [10]. From there it just remains to verify via analysis that the controller so obtained indeed achieves -modulo the choice of adequate control parametersthe practical stabilization of the complete system. The two aforementioned control design steps are detailed next.

\section{B. Control of the first subsystem}

This subsystem involves the first three state variables of the system (10) and its equations are:

$$
\left\{\begin{array}{l}
\dot{x}_{1}=c_{x_{3}} x_{5}^{\star} \\
\dot{x}_{2}=s_{x_{3}} x_{5}^{\star} \\
\dot{x}_{3}=x_{4}^{\star} x_{5}^{\star}
\end{array} .\right.
$$

Let $g_{r}(t) \equiv\left[x_{r}(t), y_{r}(t), \theta_{r}(t)\right]^{\top}$ denote the reference pose at time $t$ for the skateboard body. This is an element of the Lie group $S E(2)$, like the skateboard pose $g$, and its timederivative satisfies the relation:

$$
\dot{g}_{r}(t)=\bar{R}\left(\theta_{r}\right) u_{r}
$$

with $u_{r}(t) \in \mathbb{R}^{3}$ denoting the reference pose velocity whose norm is assumed to be bounded by $M<+\infty$. Note that, unlike the skateboard, nonholonomic constraints are a priori not imposed on the reference pose. Therefore $g_{r}(t)$ characterizes the position and orientation of a reference frame that can move freely on the plane. To render the reference pose trajectory feasible for the skateboard one would have to further impose on the second component of $u_{r}$ to be identically equal to zero. Let:

$$
\tilde{g} \equiv g_{r}^{-1} \bullet g=\left[\begin{array}{c}
R\left(-\theta_{r}\right)\left[\begin{array}{l}
x-x_{r} \\
y-y_{r}
\end{array}\right] \\
\theta-\theta_{r}
\end{array}\right]
$$

denote the pose error. The time-derivative of this error along the solutions of System (11) is given by:

$$
\dot{\tilde{g}}=\left[\begin{array}{c}
c_{\tilde{g}_{3}} \\
s_{\tilde{g}_{3}} \\
x_{4}^{\star}
\end{array}\right] x_{5}^{\star}+p_{g}(\tilde{g}, t)
$$

with:

$$
p_{g}(\tilde{g}, t) \equiv-u_{r}(t)+\left[\begin{array}{c}
\tilde{g}_{2} u_{r, 3}(t) \\
-\tilde{g}_{1} u_{r, 3}(t) \\
0
\end{array}\right]
$$

and $\tilde{g}_{i}(i=1,2,3)$ denoting the $i t h$ component of $\tilde{g}$. Let $f_{g}:(\alpha, t) \longmapsto f_{g}(\alpha, t)$ denote a bounded twice-differentiable function from $\mathbb{T}^{2} \times \mathbb{R}$ to $S E(2)$, and define:

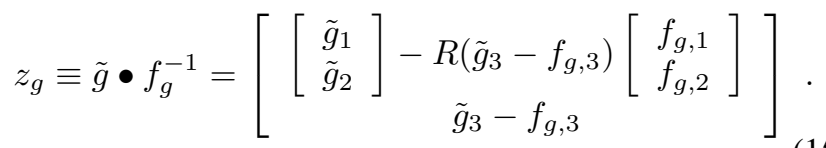

If $z_{g}$ converges to zero (i.e. the neutral element of $S E(2)$ ) then $\tilde{g}$ converges to $f_{g}$. Therefore, if $\sup _{\alpha, t}\left|f_{g}(\alpha, t)\right|$ is small then the convergence of $z_{g}$ to zero ensures that the norm of the pose error $\tilde{g}$ is ultimately small also. This suggests to determine $x_{4}^{\star}$ and $x_{5}^{\star}$ that asymptotic stabilize $z_{g}=0$. We proceed with this idea by first calculating the time-derivative of $z_{g}$. From the definition of $z_{g}$ and (13) one verifies that:

$$
\begin{aligned}
& \dot{z}_{g}=\bar{R}\left(z_{g, 3}\right) A_{g}(\alpha, t) \\
& \quad\left(H_{g}\left(x_{4}^{\star}, \alpha, t\right)\left[\begin{array}{c}
x_{5}^{\star} \\
\dot{\alpha}
\end{array}\right]-\frac{\partial f_{g}}{\partial t}+\bar{R}\left(-z_{g, 3}\right) p_{g}(\tilde{g}, t)\right)
\end{aligned}
$$

with:

$$
A_{g}(\alpha, t)=\left[\begin{array}{ccc}
1 & 0 & f_{g, 2}(\alpha, t) \\
0 & 1 & -f_{g, 1}(\alpha, t) \\
0 & 0 & 1
\end{array}\right]
$$

and:

$$
H_{g}\left(x_{4}^{\star}, \alpha, t\right)=\left[\begin{array}{cc}
c_{f_{g, 3}(\alpha, t)} & \\
s_{f_{g, 3}(\alpha, t)} & -\frac{\partial f_{g}}{\partial \alpha}(\alpha, t) \\
x_{4}^{\star} &
\end{array}\right] .
$$

Let us now assume that $x_{4}^{*}:(\alpha, t) \longmapsto x_{4}^{*}(\alpha, t)$ is also a bounded smooth function defined on $\mathbb{T}^{2} \times \mathbb{R}$. Then, (16) becomes:

$$
\begin{aligned}
\dot{z}_{g}= & \bar{R}\left(z_{g, 3}\right) A_{g}(\alpha, t) \\
& \left(\bar{H}_{g}(\alpha, t)\left[\begin{array}{c}
x_{5}^{\star} \\
\dot{\alpha}
\end{array}\right]-\frac{\partial f_{g}}{\partial t}+\bar{R}\left(-z_{g, 3}\right) p_{g}(\tilde{g}, t)\right)
\end{aligned}
$$

with:

$$
\bar{H}_{g}(\alpha, t)=\left[\begin{array}{cc}
c_{f_{g, 3}(\alpha, t)} & \\
s_{g_{g, 3}(\alpha, t)} & -\frac{\partial f_{g}}{\partial \alpha}(\alpha, t) \\
x_{4}^{\star}(\alpha, t) &
\end{array}\right] .
$$

This latter matrix-valued function already appears in the paper [26] where feedback control laws for car-like vehicles are designed with the TF approach. The key point is that it is possible to render this matrix always invertible by choosing the functions $f_{g}$ and $x_{4}^{\star}$ adequately (examples are provided later on). The determination of control inputs $x_{5}^{\star}$ and $\dot{\alpha}$ that exponentially stabilize $z_{g}=0$ is then a simple matter. Indeed, relation (19) may also be written as:

$$
\dot{z}_{g}=B_{g}\left(z_{g}, \alpha, t\right)\left[\begin{array}{c}
x_{5}^{\star} \\
\dot{\alpha}
\end{array}\right]+q_{g}(\tilde{g}, \alpha, t)
$$

with:

$$
\begin{aligned}
B_{g}\left(z_{g}, \alpha, t\right) \equiv & \bar{R}\left(z_{g, 3}\right) A_{g}(\alpha, t) \bar{H}_{g}(\alpha, t) \\
q_{g}(\tilde{g}, \alpha, t) \equiv & \bar{R}\left(z_{g_{3}, 3}\right) A_{g}(\alpha, t) \\
& \left(-\frac{\partial f_{g}}{\partial t}(\alpha, t)+\bar{R}\left(-z_{g, 3}\right) p_{g}(\tilde{g}, t)\right) .
\end{aligned}
$$


Note that $B_{g}$ is invertible $\forall\left(z_{g}, \alpha, t\right)$. Therefore, closing the loop with the control:

$$
v_{g} \equiv\left[\begin{array}{c}
x_{5}^{\star} \\
\dot{\alpha}
\end{array}\right]=-B_{g}^{-1}\left(z_{g}, \alpha, t\right)\left(K_{g} z_{g}+q_{g}(\tilde{g}, \alpha, t)\right)
$$

with $K_{g}$ a positive definite matrix, yields $\dot{z}_{g}=-K_{g} z_{g}$ and thus:

$$
\frac{d}{d t}\left|z_{g}(t)\right|^{2}=-2 z_{g}^{\top}(t) K_{g} z_{g}(t)(\leq 0)
$$

This latter relation in turn implies the exponential stability of $z_{g}=0$. A slightly more sophisticated control yielding the same relation, and thus the same rate of convergence of $\left|z_{g}\right|$ to zero, is:

$$
v_{g} \equiv\left[\begin{array}{c}
x_{5}^{\star} \\
\dot{\alpha}
\end{array}\right]=-\frac{\left(W_{g}^{-1} B_{g}^{\top} z_{g} z_{g}^{\top}+\epsilon_{g} B_{g}^{-1}\right)\left(K_{g} z_{g}+q_{g}\right)}{z_{g}^{\top} B_{g} W_{g}^{-1} B_{g}^{\top} z_{g}+\epsilon_{g}}
$$

with $W_{g}$ a symmetric positive matrix and $\epsilon_{g}$ a small positive number chosen different from zero to guarantee that the control is well defined when $z_{g}=0$. The interest of this second control is that $v_{g}^{\top} W_{g} v_{g}$ is minimized at each timeinstant when $\epsilon_{g}=0$. This is useful to penalize $\left|x_{5}^{\star}\right|$ more than the norm of $\dot{\alpha}$.

\section{Control of the second subsystem}

This subsystem involves the last four state variables of the system (10). Its equations are:

$$
\left\{\begin{array}{l}
\dot{x}_{4}=u_{1} \\
\dot{x}_{5}=x_{4} u_{2} \\
\dot{x}_{6}=u_{2} \\
\dot{x}_{7}=x_{6}
\end{array}\right.
$$

and the control inputs $u_{1}$ and $u_{2}$ are in charge of making $x_{i}$ $(i=4,5,6)$ track its desired value $x_{i}^{\star}$, with $x_{6}^{\star}$ standing for the desired time-derivative of $x_{7}$. The desired values $x_{4}^{\star}$ and $x_{5}^{\star}$ have supposedly been determined previously. Recall that $x_{7}=$ $\bar{\psi} \equiv-\frac{J_{b}}{m}\left(\left(1+\frac{J}{J_{b}}\right) \theta+\psi\right)$ so that $x_{6}=\dot{\bar{\psi}}=-\frac{J_{b}}{m}\left(\left(1+\frac{J}{J_{b}}\right) \dot{\theta}+\dot{\psi}\right)$. In the case where one is not interested in actively controlling the momentum bar angle $\psi$ (to keep it bounded, for instance) a possibility, among others, is to set $x_{6}^{\star}=0$. This means that the momentum bar angular velocity $\dot{\psi}$ will ideally be equal to $-\left(1+\frac{J}{J_{b}}\right) \dot{\theta}$. Another possibility is to set $x_{6}^{\star}=-k_{7} x_{7}$ with $k_{7}>0$ so that $x_{7}$ will ideally converge to zero. But this would still not ensure the boundedness of $\psi$. To achieve this latter property we propose instead to set:

$$
x_{6}^{\star}=-\frac{J_{b}}{m}\left(1+\frac{J}{J_{b}}\right) \dot{\bar{\theta}}+k_{7} \frac{J_{b}}{m} \psi
$$

with $\dot{\bar{\theta}}$ equal either to $\dot{\theta}_{r}$ or to the bounded derivative of a filtered value of $\theta$. The reason for this choice is that it yields $\dot{\psi}=-k_{7} \psi-\left(1+\frac{J}{J_{b}}\right)(\dot{\theta}-\dot{\bar{\theta}})$ when $x_{6}=x_{6}^{\star}$ and, subsequently, the boundedness of $|\psi|$ when $|\dot{\theta}-\dot{\bar{\theta}}|$ is itself bounded. It also allows for the convergence of $\psi$ to zero when this latter difference converges to zero.

From there we use the fact that the first three equations of the subsystem (25) is a 3-dimensional chained system that is left-invariant on the Lie group $\mathbb{R}^{3}$ endowed with the group operation defined by:

$$
a \star b=\left[\begin{array}{c}
a_{1}+b_{1} \\
a_{2}+b_{2}+a_{1} b_{3} \\
a_{3}+b_{3}
\end{array}\right]
$$

and the neutral element $e=[0,0,0]^{\top}$. This leads to define the error vector

$$
\xi \equiv\left[\begin{array}{c}
x_{4}^{\star} \\
x_{5}^{\star} \\
x_{6}^{\star}
\end{array}\right]^{-1} \star\left[\begin{array}{c}
x_{4} \\
x_{5} \\
x_{6}
\end{array}\right]=\left[\begin{array}{c}
\tilde{x}_{4} \\
\tilde{x}_{5}-x_{4}^{\star} \tilde{x}_{6} \\
\tilde{x}_{6}
\end{array}\right]
$$

with $\tilde{x}_{i} \equiv x_{i}-x_{i}^{\star}$, whose time-derivative satisfies the equation

$$
\dot{\xi}=\left[\begin{array}{c}
u_{1} \\
\xi_{1} u_{2} \\
u_{2}
\end{array}\right]+p_{\xi}\left(\xi, x_{4}^{\star}, \dot{x}_{4,5,6}^{\star}\right)
$$

with:

$$
p_{\xi}\left(\xi, x_{4}^{\star}, \dot{x}_{4,5,6}^{\star}\right) \equiv\left[\begin{array}{c}
-\dot{x}_{4}^{\star} \\
-\dot{x}_{5}^{\star}+x_{4}^{\star} \dot{x}_{6}^{\star}-\dot{x}_{4}^{\star} \xi_{3} \\
-\dot{x}_{6}^{\star}
\end{array}\right] .
$$

The calculation of this latter vector involves the calculation of the derivatives of $x_{i}^{\star}(i=4,5,6)$. From the expression of $x_{6}^{\star}$ (relation (26))

$$
\dot{x}_{6}^{\star}=-\frac{J_{b}}{m}\left(1+\frac{J}{J_{b}}\right) \ddot{\ddot{\theta}}+k_{7} \frac{J_{b}}{m} \dot{\psi} .
$$

As for $\dot{x}_{5}^{\star}$ it has to be calculated from the expression of $x_{5}^{\star}$ given in relation (22) or (24), depending on the chosen control law. This calculation does not pose particular difficulties but it is tedious. For this reason, it is not reported here. The important fact is that it does not involve the control inputs $u_{1}$ and $u_{2}$ whose calculations, as we will see next, depend on the beforehand calculation of $p_{\xi}$, and thus of $\dot{x}_{5}^{\star}$.

Let us now proceed with the practical stabilization of $\xi$ about zero. Let $h: \beta \longmapsto h(\beta)$ denote a differentiable function from $\mathbb{T}$ to $\mathbb{R}^{3}$ and define:

$$
z_{\xi} \equiv \xi \star h^{-1}=\left[\begin{array}{c}
\left(\xi_{1}-h_{1}\right) \\
\left(\xi_{2}-h_{2}\right)-h_{3}\left(\xi_{1}-h_{1}\right) \\
\left(\xi_{3}-h_{3}\right)
\end{array}\right] .
$$

The convergence of $z_{\xi}$ to zero then yields the convergence of $\xi$ to $h(\beta)$ and thus the ultimate boundedness of $|\xi|$ and $\left|\tilde{x}_{i}\right|$ $(i=4,5,6)$ by small values if $|h|$ is itself bounded by a small value. By direct calculation, or by using classical differential calculus on a Lie group (see [10], for instance), one verifies that:

$$
\dot{z}_{\xi}=B_{\xi}\left(z_{\xi}, \beta\right)\left[\begin{array}{c}
u_{1} \\
u_{2} \\
\dot{\beta}
\end{array}\right]+q_{\xi}\left(p_{\xi}, \beta\right)
$$

with:

$$
\begin{aligned}
& B_{\xi}\left(z_{\xi}, \beta\right) \equiv A_{\xi}(\xi, \beta) H_{\xi}(\beta), \\
& A_{\xi}\left(z_{\xi}, \beta\right) \equiv\left[\begin{array}{ccc}
1 & 0 & 0 \\
-h_{3}(\beta) & 1 & z_{\xi, 1} \\
0 & 0 & 1
\end{array}\right], \\
& H_{\xi}(\beta)=\left[\begin{array}{ccc}
1 & 0 & \\
0 & h_{1}(\beta) & -\frac{\partial h}{\partial \beta}(\beta) \\
0 & 1 &
\end{array}\right],
\end{aligned}
$$


and:

$$
q_{\xi}\left(p_{\xi}, \beta\right) \equiv\left[\begin{array}{ccc}
1 & 0 & 0 \\
-h_{3}(\beta) & 1 & 0 \\
0 & 0 & 1
\end{array}\right] p_{\xi} .
$$

An adequate choice of the function $h$ ensures that the matrix $H_{\xi}(\beta)$ is invertible $\forall \beta$. This in turn ensures that $B_{\xi}\left(z_{\xi}, \beta\right)$ is invertible for any $\left(z_{\xi}, \beta\right)$. It is then simple to derive control inputs $u_{1}, u_{2}$ and $\dot{\beta}$ that exponentially stabilizes $z_{\xi}=0$. Take, for instance:

$$
v_{\xi} \equiv\left[\begin{array}{c}
u_{1} \\
u_{2} \\
\dot{\beta}
\end{array}\right]=-B_{\xi}^{-1}\left(z_{\xi}, \beta\right)\left(K_{\xi} z_{\xi}+q_{\xi}\left(p_{\xi}, \beta\right)\right)
$$

with $K_{\xi}$ a positive matrix, or:

$$
v_{\xi} \equiv\left[\begin{array}{c}
u_{1} \\
u_{2} \\
\dot{\beta}
\end{array}\right]=-\frac{\left(W_{\xi}^{-1} B_{\xi}^{\top} z_{\xi} z_{\xi}^{\top}+\epsilon_{\xi} B_{\xi}^{-1}\right)\left(K_{\xi} z_{\xi}+q_{\xi}\right)}{z_{\xi}^{\top} B_{\xi} W_{\xi}^{-1} B_{\xi}^{\top} z_{\xi}+\epsilon_{\xi}}
$$

with $W_{\xi}$ a positive definite matrix and $\epsilon_{\xi}$ a small positive number. Either one of these feedback controls yields the closed-loop equation:

$$
\frac{d}{d t}\left|z_{\xi}\right|^{2}=-2 z_{\xi}^{\top} K_{\xi} z_{\xi}(\leq 0) .
$$

\section{Stability analysis of the complete system}

The control design exposed in the previous subsection is based on the separate control of subsystems (11) and (25), and its effectiveness relies on the possibility of assigning arbitrary desired values $\left(x_{4}^{*}, x_{5}^{*}\right)$ to $\left(x_{4}, x_{5}\right)$. However, the control derived for the second subsystem only ensures the practical stability of $\left(x_{4}^{*}, x_{5}^{*}\right)$ for $\left(x_{4}, x_{5}\right)$. This approximation justifies a rigorous complementary analysis whose conclusion, which may be viewed as the main result of this paper, is summarized by the following proposition

Proposition 4.1: Let $f_{g}: \mathbb{T}^{2} \times \mathbb{R} \rightarrow S E(2)$ and $x_{4}^{\star}$ : $\mathbb{T}^{2} \times \mathbb{R} \rightarrow \mathbb{R}$ denote bounded functions of class $\mathcal{C}^{2}$ and $\mathcal{C}^{1}$ respectively, with the partial derivatives $\frac{\partial f_{g}}{\partial \alpha}$ and $\frac{\partial f_{g}}{\partial t}$ of $f_{g}$ being also bounded. Define:

$$
\varepsilon_{f}=: \max \left\{\sup _{\alpha, t}\left|f_{g}(\alpha, t)\right|, \sup _{\alpha, t}\left|x_{4}^{\star}(\alpha, t)\right|\right\} .
$$

Let $h: \mathbb{T} \rightarrow \mathbb{R}$ denote a function of class $\mathcal{C}^{1}$ and denote $\varepsilon_{h}=: \max _{\beta}|h(\beta)|$. Assume that:

1) $f_{g}$ and $x_{4}^{\star}$ are chosen so that $\bar{H}_{g}(\alpha, t)$ is invertible $\forall(\alpha, t)$ and $\inf _{\alpha, t}\left|\operatorname{det}\left(\bar{H}_{g}(\alpha, t)\right)\right|>0$,

2) $h$ is chosen so that $H_{\xi}(\beta)$ is invertible $\forall \beta$.

Then, there exists $\bar{\varepsilon}_{h}>0$ such that for any $\varepsilon_{h} \leq \bar{\varepsilon}_{h}$, the application of the dynamic feedback control $\left(u_{1}, u_{2}\right)$ defined by either (32) or (33) grants the following properties:

- The pose tracking error $|\tilde{g}|$ is ultimately bounded along the solutions of the controlled system by a value independent of the initial conditions.

- This bound is itself upper bounded by $\varepsilon_{f}$ as $\varepsilon_{h}$ tends to zero.

The proof of this result is given in the Appendix.
This proposition indicates that arbitrarily small pose tracking errors can be obtained by using functions $f_{g}$ and $h$ calculated with $\varepsilon_{f}$ and $\varepsilon_{h}$ chosen "small enough". This property does not rely on the feasibility of the reference trajectory and is coherent with the use of large control gains and high manoeuvring frequencies when non-feasible reference trajectories must be precisely tracked. In practice a compromise thus has to be found between high precision tracking in all situations and limitation of the control energy expenditure.

\section{E. Associated transverse functions}

The implementation of the proposed feedback control laws relies on the selection and use of functions $f_{g}, x_{4}^{\star}$ and $h$ that satisfy the assumptions of Proposition 4.1 (the transversality conditions specified in items 1. and 2. in particular). Such functions are pointed out next.

As already noticed System (11) coincides with a kinematic model of a car-like vehicle. The following functions $f_{g}$ and $x_{4}^{\star}$, defined on $\mathbb{T}^{2} \times \mathbb{R}$, were first considered in [26]:

$$
f_{g}=\left(f_{1}, f_{2}, f_{3}\right)^{\top}, \quad x_{4}^{*}=f_{4}+\bar{\eta}_{r},
$$

with:

$$
\begin{gathered}
f(\alpha, t)=\Psi\left(f_{c}(\alpha), \bar{\eta}_{r}(t)\right), \\
f_{c, 1} \\
f_{c}(\alpha)=\left[\begin{array}{c}
\left.f_{c, 4}+\bar{\eta}_{r}\right)=\left[\begin{array}{c}
f_{c, 1} / 2 \\
\operatorname{atan}\left(f_{c, 3}+\bar{\eta}_{r} f_{c, 1}\right) \\
\frac{f_{c, 2}+\bar{\eta}_{r}}{\left(1+\left(f_{c, 3}+\bar{\eta}_{r} f_{c, 1}\right)^{2}\right)^{3 / 2}}-\bar{\eta}_{r}
\end{array}\right], \\
\epsilon_{1}\left(s_{\alpha_{1}}+\mu\right)+\epsilon_{3}\left(s_{\alpha_{2}}+\mu\right) \\
\epsilon_{2} c_{\alpha_{1}} \\
\frac{\epsilon_{1} \epsilon_{2}}{4} s_{2 \alpha_{1}}-\epsilon_{4} c_{\alpha_{2}} \\
\frac{\epsilon_{1}^{2} \epsilon_{2}}{6}\left(s_{\alpha_{1}}\right)^{2} c_{\alpha_{1}}-\frac{\epsilon_{3} \epsilon_{4}}{4} s_{2 \alpha_{2}}-\epsilon_{1} \epsilon_{4} s_{\alpha_{1}} c_{\alpha_{2}}
\end{array}\right],
\end{gathered}
$$

$\alpha=\left[\alpha_{1}, \alpha_{2}\right]^{\top}, \mu=0$ or $\mu=1 . \bar{\eta}_{r}$ is a bounded time function of class $\mathcal{C}^{1}$ whose derivative is also bounded. These functions satisfy the assumptions of Proposition 4.1 (proof in [26]). In the case where $\bar{\eta}_{r} \equiv 0, f$ and $x_{4}^{\star}$ are functions of $\alpha$ only, and thus do not depend on the exogenous time variable. However, this choice is not best suited to the tracking of reference trajectories. Indeed, the role of $\bar{\eta}_{r}$ is to precompensate for the reference path curvature when $u_{r, 1}(t) \neq 0$ and $u_{r, 2}(t) \approx 0$. In this case it should not be very different from $\eta_{r}(t)=\dot{\theta}_{r}(t) / u_{r, 1}(t)$. Its value can be chosen close to zero otherwise. For the sake of completeness let us mention that $f_{c}(\alpha)$ is a transverse function for the 4-dimensional chained system. The rank of the matrix $H_{g}$ does not depend on the choice of $\bar{\eta}_{r}(t)$, nor on the choice of $\mu$. However, to ensure that $\bar{H}_{g}(\alpha, t) \equiv H_{g}\left(x_{4}^{\star}(\alpha, t), \alpha, t\right)$ is invertible $\forall(\alpha, t)$ the parameters $\epsilon_{1,2,3,4}$ must be different from zero and satisfy the following inequalities:

$$
\left|\epsilon_{3}\right|>\frac{4}{3}\left|\epsilon_{1}\right|, \quad\left|\epsilon_{4}\right|>\frac{\left|\epsilon_{2}\right|}{2\left(\left(3 /\left|\epsilon_{1}\right|\right)-\left(4 /\left|\epsilon_{3}\right|\right)\right)}>0 .
$$

As for the parameter $\mu$, it can be set equal to zero to center $f_{c}(\alpha)$ and $f(\alpha, t)$ about the zero vector, or equal to one to have the zero vector belong to the image of $f_{c}(\alpha)$ and $f(\alpha, t)$ (for $\alpha_{1}=\alpha_{2}=-\pi / 2$ ). As explained in [26], this latter choice 
is of interest to allow for the asymptotic stabilization of zero tracking errors when $\left|u_{r, 1}(t)\right|>0$ and $u_{r, 2} \equiv 0$ (i.e. when the reference frame trajectory is feasible for a nonholonomic carlike vehicle). The parameters $\epsilon_{1}$ and $\epsilon_{3}$ should then be of the same sign as $u_{r, 1}(t)$ so that $\alpha_{1}$ and $\alpha_{2}$ can converge to $-\pi / 2$ when $z_{g}$ converges to zero. The simultaneous convergence of $z_{g}$ and $f(\alpha, t)$ to zero then yields the convergence of $\tilde{g}$ to zero. Nevertheless, independently of the values taken by these angles, the norm of the function $f$, and thus of $f_{g}$, is commensurable with the size of the parameters $\epsilon_{1,2,3,4}$. This norm can thus be as small as desired by choosing the absolute values of these parameters small enough (but different from zero).

Concerning the function $h$ invoked for the control of the second subsystem, a possible (bounded) transverse function that renders $H_{\xi}(\beta)$ invertible $\forall \beta$ is given by ( $[10$, Sec. VII.C]):

$$
h(\beta)=\left[\begin{array}{cc}
\epsilon_{5} & s_{\beta} \\
\epsilon_{5} \epsilon_{6} & s_{2 \beta} \\
\epsilon_{6} & c_{\beta}
\end{array}\right]
$$

with $\epsilon_{5}$ and $\epsilon_{6}$ denoting non-zero real numbers. Indeed, one easily verifies that this function yields $\operatorname{det}\left(H_{\xi}(\beta)\right)=\epsilon_{5} \epsilon_{6} / 2$.

\section{Simulation Results}

For this simulation the skateboard parameters are $m=$ $50 \mathrm{~kg}, l=0.3 \mathrm{~m}, J=0.1 \mathrm{~kg} \cdot \mathrm{m}^{2}$, and $J_{b}=3 \mathrm{~kg} \cdot \mathrm{m}^{2}$. The reference frame moves from its initial pose with the velocities indicated in the following chart.

\begin{tabular}{|c|c|}
\hline $\mathrm{t}(\mathrm{sec}) \in$ & $u_{r}(t)^{\top}$ \\
\hline \hline$[0,5)$ & {$[0,0,0]$} \\
\hline$[5,9)$ & {$[1.2,0,0.2(t-5)]$} \\
\hline$[9,13)$ & {$[1.2,0,0.8-0.2(t-9)]$} \\
\hline$[13,16)$ & {$[1.2,0,0.3(2 / 1.75) \pi \cos ((2 / 1.75) \pi(t-13))]$} \\
\hline$[16,26)$ & {$[0,0,0.3]$} \\
\hline$[26,29)$ & {$[0.6(t-26), 0,-0.08(t-26)]$} \\
\hline$[29,32)$ & {$[1.8,0,-0.24-0.08(t-29)]$} \\
\hline$[32,37)$ & {$[0,-0.1(t-32), 0]$} \\
\hline$[37,42)$ & {$[0,-0.5,0]$} \\
\hline$[42,47)$ & {$[0,-0.5+0.1(t-42), 0]$} \\
\hline$[47,60)$ & {$[-0.2(t-47), 0,-0.06(t-47)]$} \\
\hline$[60,70)$ & {$[0,0,0]$} \\
\hline
\end{tabular}

The reference frame trajectory $g_{r}(t)$ is then obtained by integration of both members of (12). This trajectory is visualized in Fig 2. Note the existence of velocity discontinuities at a certain number of time-instants $(\mathrm{t}=5,16,32,60)$ that the control has to overcome. Note also that the reference frame is at rest on the time-intervals $[0,5)$ and $[60,70)$ corresponding to fixed-point stabilization phases, and that the reference frame trajectory is not feasible for the skateboard kinematics on the time-intervals $[16,26)$ and $[29,47)$ because of pure rotation motion (when $u_{r, 1}(t)=u_{r, 2}(t)=0, u_{r, 3}(t) \neq 0$ ) and lateral motion (when $u_{r, 2}(t) \neq 0$ ) that violate nonholonomic constraints, since the driving wheels' angle $|\phi|$ is always smaller than $\pi / 2$. Accelerating along a straight line (i.e. $\dot{v}_{x} \neq 0$ with $\eta=0$ ) is not feasible either. As a matter

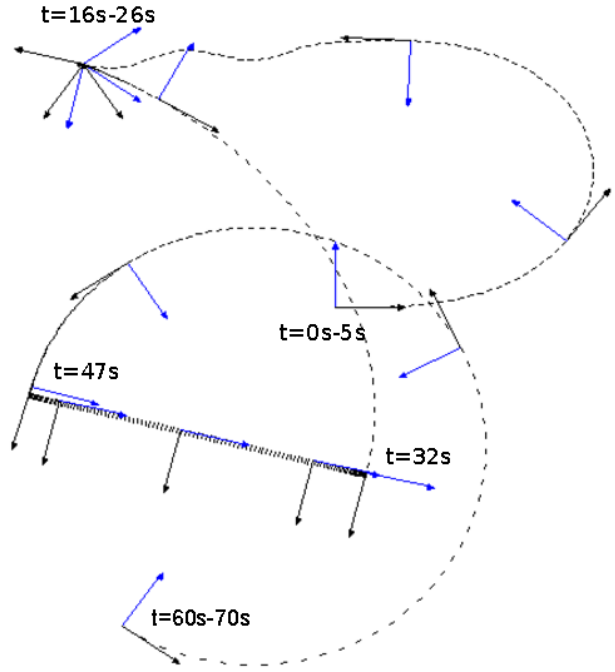

Fig. 2. Reference frame trajectory

of fact, even moving with a constant velocity $v_{x}$ along a straight line is not possible when viscous friction terms that tend to slow down the skateboard are added to the simulated system's dynamics. For the present simulation a friction force $F_{\text {friction }}=-25 c_{\phi} \quad v_{x}$ in the direction of the skateboard's longitudinal axis is added to the system's dynamics, but is not taken into account at the control design level so as to test the robustness of the control w.r.t. this unmodeled term. The necessity of creating a propulsion force that compensates for friction also explains the undulatory motion performed by skateboard riders to maintain an approximately constant longitudinal velocity along a desired direction.

The feedback control used for the simulation is given by (33) for the calculation of $\left(u_{1}, u_{2}, \dot{\beta}\right)$, complemented with (24) for the calculation of $\left(x_{5}^{\star}, \dot{\alpha}\right)$. The control parameters are set to $K_{\xi}=5 I_{d}, K_{g}=I_{d}, \epsilon_{\xi}=\epsilon_{g}=0.01, W_{\xi}=\operatorname{Diag}\{1,1,0.01\}$, $W_{g}=\operatorname{Diag}\{1,0.01,0.01\}$. The transverse function parameters are:

$$
\begin{aligned}
& \left(\epsilon_{2}, \epsilon_{4}, \epsilon_{5}, \epsilon_{6}\right)=(2,0.64,0.6,0.6), \\
& \left(\epsilon_{1}, \epsilon_{3}, \mu\right)=\left\{\begin{array}{c}
(0.15,0.8,0) \text { when }\left|u_{r, 1}(t)\right|<0.1 \mathrm{~m} / \mathrm{s} \\
\left(0.15 \operatorname{sgn}\left(u_{r, 1}(t)\right), 0.8 \operatorname{sgn}\left(u_{r, 1}(t)\right), 1\right) \\
\text { otherwise }
\end{array} .\right.
\end{aligned}
$$

The term $\bar{\eta}_{r}(t)$ involved in the expression (35) of the transverse function $f(\alpha, t)$ is calculated according to $\ddot{\bar{\eta}}_{r}=-2 \dot{\bar{\eta}}_{r}-$ $\left(\bar{\eta}_{r}-\eta^{\star}\right)$ with $\eta^{\star}=\dot{\theta}_{r} u_{r, 1} /\left(u_{r, 1}^{2}+0.01\right)$. As for the term $\dot{\bar{\theta}}$ involved in the expression (26) of $x_{6}^{\star}$, a filtered value of $\theta$ calculated according to $\dot{\bar{\theta}}=3 \tanh (2(\theta-\bar{\theta}))$ is used. The gain associated with the effort to keep the momentum bar angle bounded is set to $k_{7}=10$.

Initial poses of the reference frame and of the skateboard are $g_{r}=[0,0,0]^{\top}$ and $g=[1,-1,0]^{\top}$ respectively.

Figure 3(a) shows the skateboard position trajectory (blue curve) superposed to the reference frame position trajectory (black dotted curve). It illustrates in particular the manoeuvres performed by the skateboard during phases when the reference 


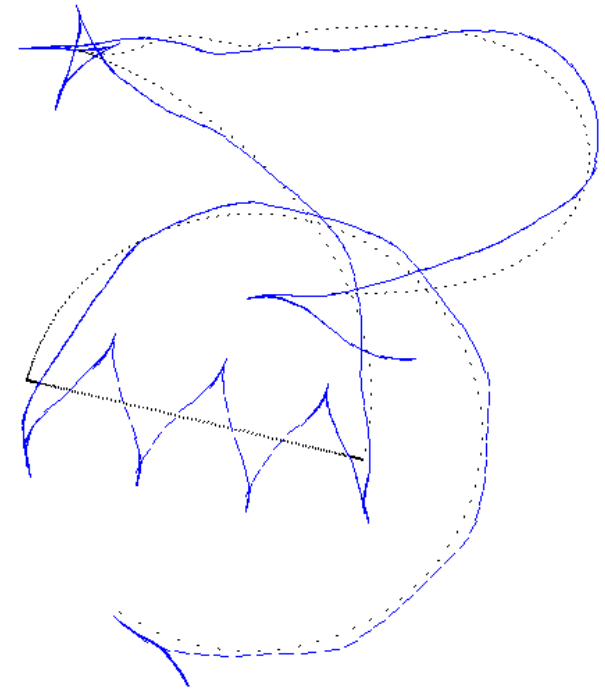

(a)

Fig. 3. Reference frame and skateboard position trajectories

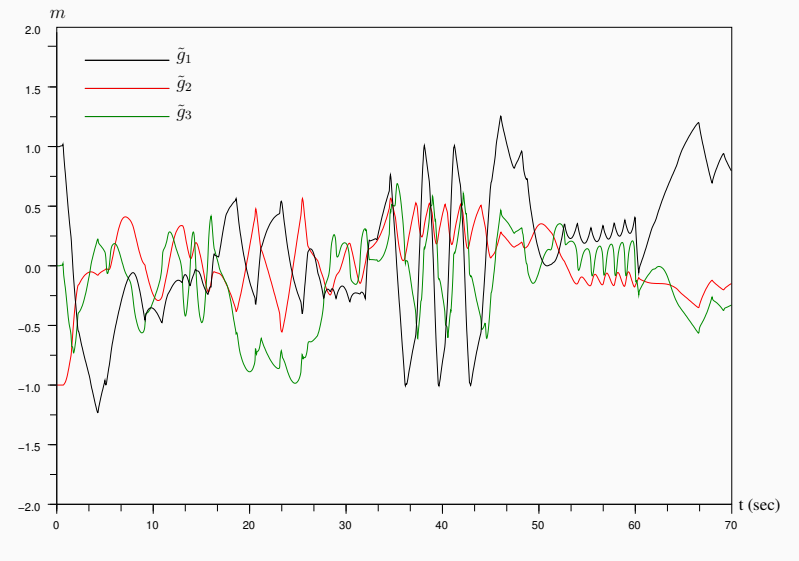

(b)

Fig. 3. Pose errors vs. time

frame motion is not compatible with the nonholonomic skateboard kinematics. Figure 3(b) shows the time-evolution of the pose error coordinates. Figure 3(c) shows the time-evolution of the momentum bar angle $\psi$ and of the driving wheels angle $\phi$. The self-synchronization of these angles creating undulatory motion, in particular during the circling phase $(t \in[47,60)$ ) when the skateboard smoothly accelerates until the final brisk stop, is noticeable. The boundedness of the momentum bar angle is also worth noticing. Finally, Figs 3(d) and 3(e) show the values taken by the torque $\tau_{\psi}$ applied to the momentum bar and by the driving wheels angle velocity $\dot{\phi}$. Note the strong impulsive values taken by these control inputs at specific timeinstants. A way to reduce these values in order to comply with the capacities of small torque motors consists in saturating the control inputs $u_{1}$ and $u_{2}$. A way to implement this saturation is

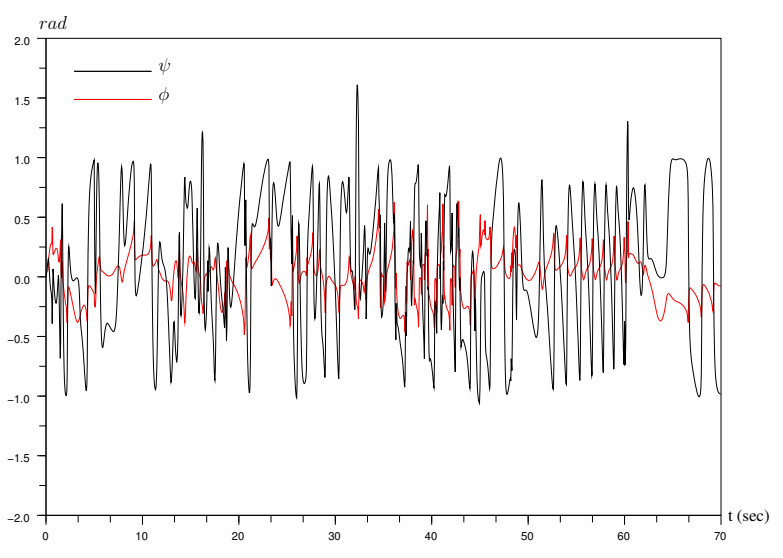

(c)

Fig. 3. Momentum bar and driving wheels angles vs. time

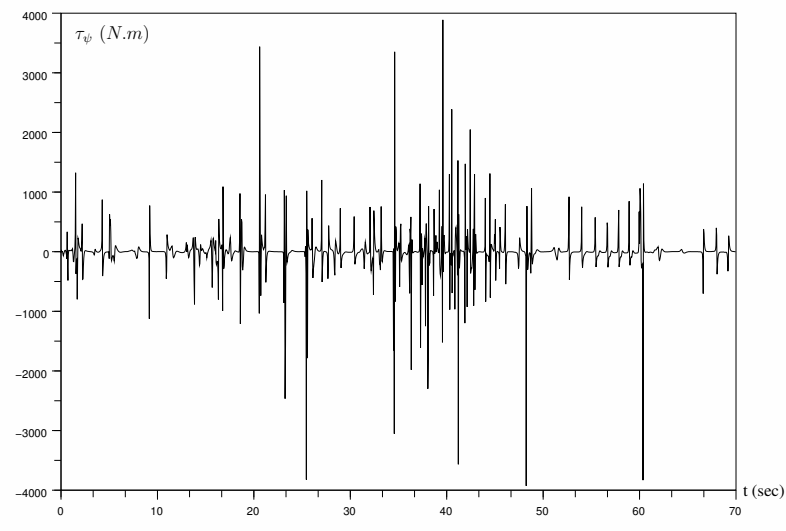

(d)

Fig. 3. Torque applied to the momentum bar vs. time

to multiply the original input vector $\left[u_{1}, u_{2}, \dot{\beta}\right]^{\top}$ by a reduction gain $\tau_{\max } /\left|\tau_{\psi}^{c}\right|$, with $\tau_{\psi}^{c}=-m\left(1+(J / m) \eta^{2}\right) u_{2}-J u_{1} v_{x}$ denoting the momentum bar angle calculated from the model equations (see equations (7) and (8)) and $\tau_{\max }$ denoting the allowed maximal torque intensity, whenever this gain is smaller than one. The application of this saturation procedure with $\tau_{\max }=300 \mathrm{~N}$. $m$ yields the simulation results reported on Figures 4(a)-4(e).

From these figures one can observe a slight deterioration of the skateboard tracking performance, especially during nonholonomic motion phases that involve a succession of accelerating and decelerating manoeuvres. This is related to the less energetic behaviour associated with drastic torque saturation. Nevertheless trajectory tracking is still performed satisfactorily, which accounts for the robustness of the control w.r.t. this type of modification. For a better visual rendering of the skateboard motion interested readers are invited to 


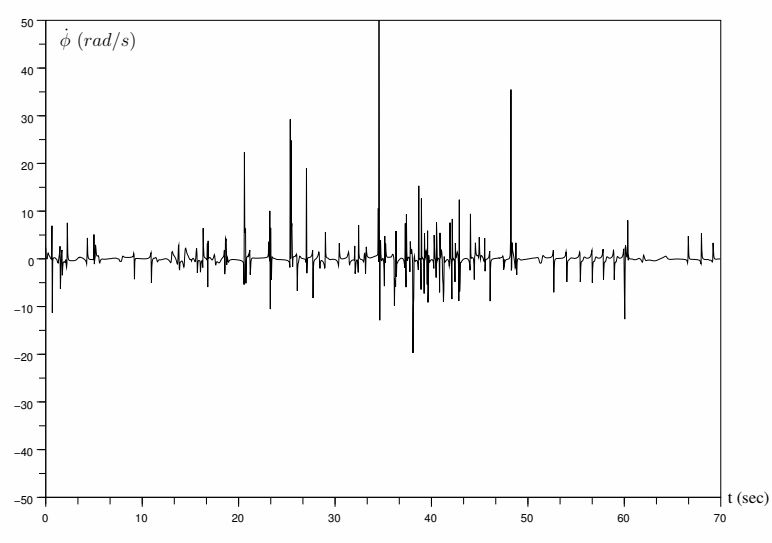

(e)

Fig. 3. Driving wheels angle velocity vs. time

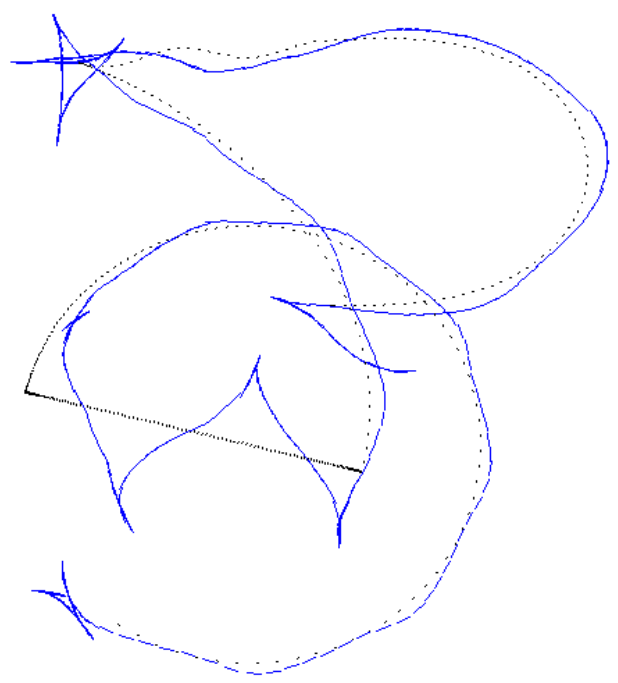

(a)

Fig. 4. Reference frame and skateboard position trajectories

visit the website www.transversefunction.com and view the corresponding simulation video.

\section{CONCLUSION}

A feedback control solution for the motorized skateboard has been derived by combining the Transverse Function control approach with backstepping. Motion planning for this system has been addressed in several previous articles, but we are not aware of the existence of other published feedback solutions. A complementary asset of the proposed approach is that it allows for the practical stabilization of arbitrary (not necessarily feasible) pose trajectories in Cartesian space, thus greatly simplifying the motion planning issue. The approach is validated by simulations, some of which incorporate unmodeled dynamic effects such as friction and torque saturations to illustrate robustness properties of the proposed control laws.

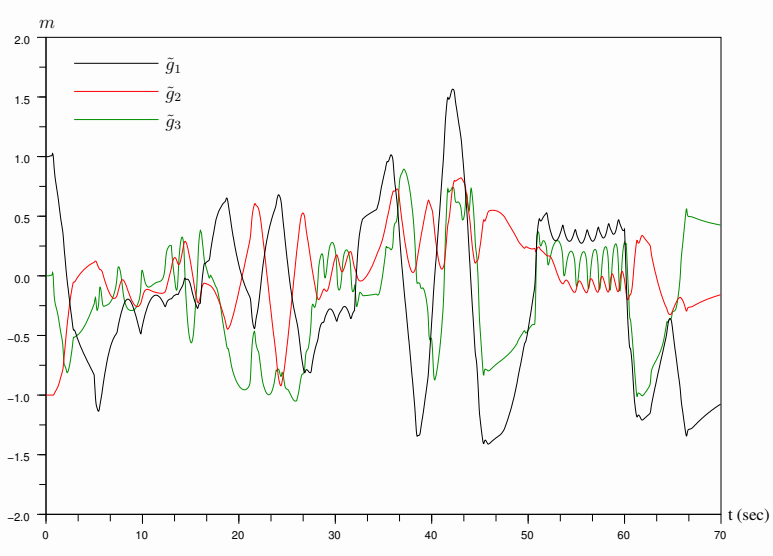

(b)

Fig. 4. Pose errors vs. time

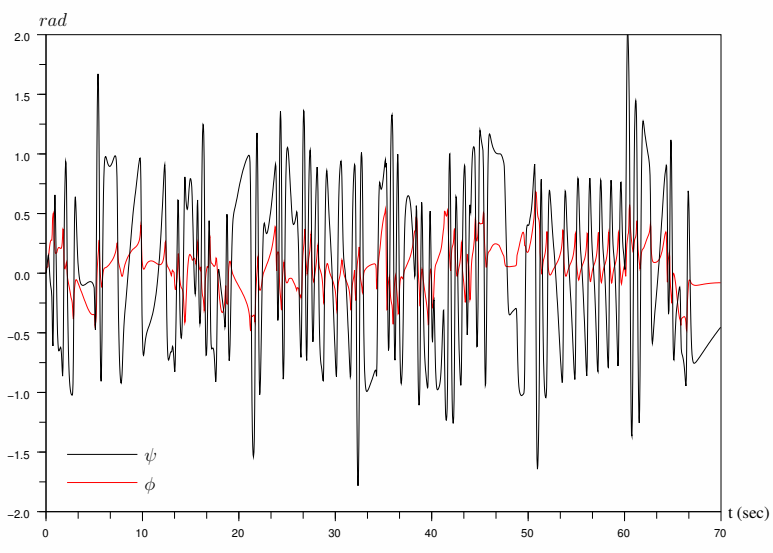

(c)

Fig. 4. Momentum bar and driving wheels angles vs. time

APPENDIX: PROOF OF PROPOSITION 4.1

We first show that the solutions of the controlled system are defined for any time $t \geq 0$. Due to the non-coincidence of $x_{5}$ and $x_{5}^{\star}$ the time derivative of $\tilde{g}$ along the solutions to System (10) is given by (compare with (13)):

$\dot{\tilde{g}}=\left[\begin{array}{c}c_{\tilde{g}_{3}} \\ s_{\tilde{g}_{3}} \\ x_{4}\end{array}\right] x_{5}+p_{g}(\tilde{g}, t)=\left[\begin{array}{c}c_{\tilde{g}_{3}} \\ s_{\tilde{g}_{3}} \\ x_{4}\end{array}\right]\left(x_{5}^{\star}+\tilde{x}_{5}\right)+p_{g}(\tilde{g}, t)$ 


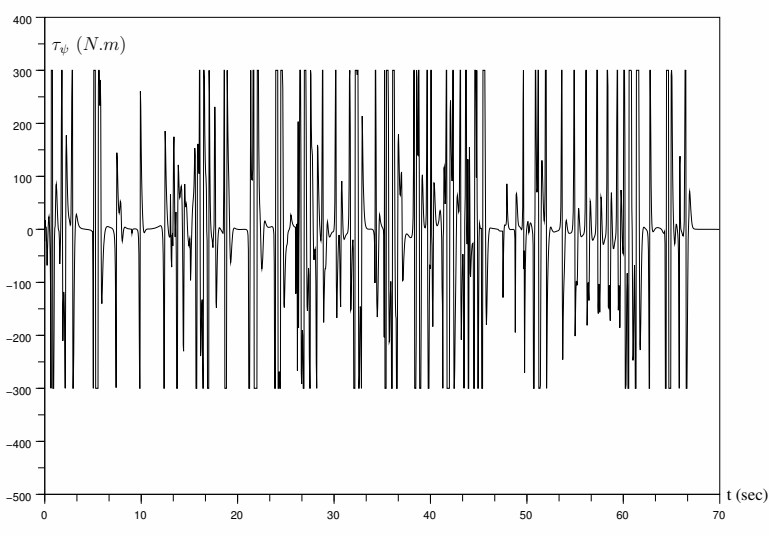

(d)

Fig. 4. Torque applied to the momentum bar vs. time

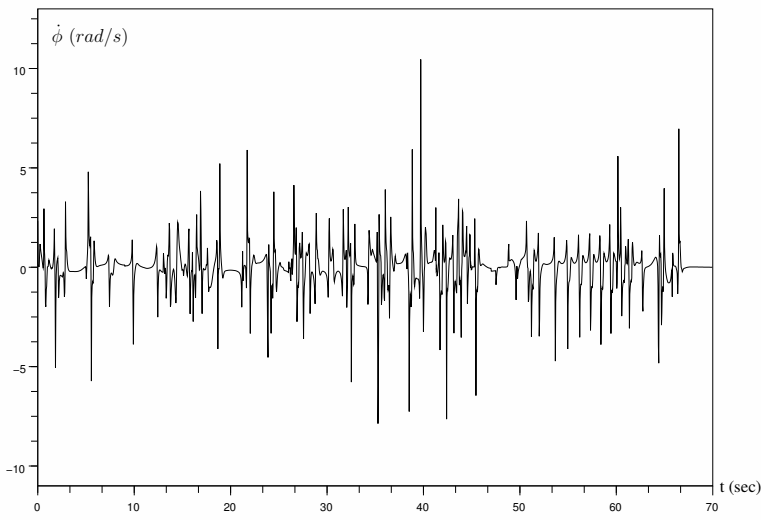

(e)

Fig. 4. Driving wheels angle velocity vs. time

with $\tilde{x}_{5} \equiv x_{5}-x_{5}^{\star}$. The equation (16) characterizing the timeevolution $z_{g}$ has in turn to be corrected into:

$$
\begin{aligned}
\dot{z}_{g}= & \bar{R}\left(z_{g, 3}\right) A_{g}(\alpha, t) \\
& \left(H_{g}\left(x_{4}, \alpha, t\right)\left[\begin{array}{c}
x_{5} \\
\dot{\alpha}
\end{array}\right]-\frac{\partial f_{g}}{\partial t}+\bar{R}\left(-z_{g, 3}\right) p_{g}(\tilde{g}, t)\right) \\
= & \bar{R}\left(z_{g, 3}\right) A_{g}(\alpha, t) \\
& \left(H_{g}\left(x_{4}^{\star}, \alpha, t\right)\left[\begin{array}{c}
x_{5}^{\star} \\
\dot{\alpha}
\end{array}\right]-\frac{\partial f_{g}}{\partial t}+\bar{R}\left(-z_{g, 3}\right) p_{g}(\tilde{g}, t)\right) \\
& +\bar{R}\left(z_{g, 3}\right) A_{g}(\alpha, t) \Delta,
\end{aligned}
$$

with:

$$
\Delta=H_{g}\left(x_{4}, \alpha, t\right)\left[\begin{array}{c}
x_{5} \\
\dot{\alpha}
\end{array}\right]-H_{g}\left(x_{4}^{\star}, \alpha, t\right)\left[\begin{array}{c}
x_{5}^{\star} \\
\dot{\alpha}
\end{array}\right] .
$$

The arguments of the function $\Delta$ are omitted to lighten the notation. From (41), one obtains (compare with (20)):

$$
\dot{z}_{g}=B_{g}(\tilde{g}, \alpha, t)\left[\begin{array}{c}
x_{5}^{\star} \\
\dot{\alpha}
\end{array}\right]+q_{g}(\tilde{g}, \alpha, t)+\bar{R}\left(z_{g, 3}\right) A_{g}(\alpha, t) \Delta .
$$

Closing the loop with $v_{g}$ defined by (22) or (24) yields, in view of (23):

$$
\frac{d}{d t}\left|z_{g}\right|^{2}=-2 z_{g}^{\top} K_{g} z_{g}+z_{g}^{\top} \bar{R}\left(z_{g, 3}\right) A_{g}(\alpha, t) \Delta .
$$

Let us now consider the expression of $\Delta$. One has:

$$
\begin{aligned}
\Delta= & \left(H_{g}\left(x_{4}, \alpha, t\right)-H_{g}\left(x_{4}^{\star}, \alpha, t\right)\right)\left[\begin{array}{c}
x_{5} \\
\dot{\alpha}
\end{array}\right] \\
& \left.+H_{g}\left(x_{4}^{\star}, \alpha, t\right)\right)\left[\begin{array}{c}
\tilde{x}_{5} \\
0
\end{array}\right],
\end{aligned}
$$

and it follows from the expression (18) of $H_{g}$ that

$$
\Delta=\tilde{x}_{5}\left[\begin{array}{c}
c f_{g, 3} \\
s f_{g, 3} \\
x_{4}^{\star}
\end{array}\right]+\left[\begin{array}{c}
0 \\
0 \\
\tilde{x}_{4} \tilde{x}_{5}
\end{array}\right]+\left[\begin{array}{c}
0 \\
0 \\
\tilde{x}_{4} x_{5}^{\star}
\end{array}\right] .
$$

Therefore:

$$
|\Delta| \leq\left|\tilde{x}_{5}\right|\left(1+\left|x_{4}^{\star}\right|+\left|\tilde{x}_{4}\right|\right)+\left|\tilde{x}_{4}\right|\left|x_{5}^{\star}\right| .
$$

Let us derive upper bounds for all the terms in the right-hand side of the above expression. It follows from (27) and (30) that:

$\tilde{x}_{4,5,6}=z_{\xi}+h(\beta)+\left(h_{3}(\beta) z_{\xi, 1}+x_{4}^{\star}(\alpha, t)\left(z_{\xi, 3}+h_{3}(\beta)\right)\right) e_{2}$

with $e_{2}=(0,1,0)^{\top}$. Using the assumed boundedness of $\left|f_{g}\right|,\left|x_{4}^{\star}\right|$, and $|h|$, the above equality then implies that ${ }^{1}$ :

$$
\tilde{x}_{4,5,6}=\mathrm{O}\left(\left|z_{\xi}\right|+1\right)
$$

Let us now derive an upper bound for $\left|x_{5}^{\star}\right|$. It follows from (1) and (15) that:

$$
\tilde{g}=z_{g} \bullet f_{g}(\alpha)=\mathrm{O}\left(\left|z_{g}\right|+1\right) .
$$

Using (14) and the assumed boundedness of $\left|u_{r}\right|$ this equality implies that:

$$
p_{g}=\mathrm{O}(|\tilde{g}|+1)=\mathrm{O}\left(\left|z_{g}\right|+1\right) .
$$

From this latter relation and (21), using the assumed boundedness of $\left|f_{g}\right|$ and $\left|x_{4}^{\star}\right|$ and also the assumed boundedness of the partial derivatives of $f_{g}$, one deduces that:

$$
q_{g}=\mathrm{O}\left(\left|z_{g}\right|+1\right) \text {. }
$$

The assumed positive lower bound of $\left|\operatorname{det}\left(\bar{H}_{g}(\alpha, t)\right)\right|$ implies that $B_{g}^{-1}$ is a bounded function. Therefore, from (46) and (22) (or (24)) it comes that:

$$
x_{5}^{\star}=\mathrm{O}\left(\left|z_{g}\right|+1\right), \quad \dot{\alpha}=\mathrm{O}\left(\left|z_{g}\right|+1\right) .
$$

Finally, one deduces from (43), (45) and (47) that:

$$
|\Delta| \leq K\left(\left|z_{\xi}\right|+1\right)\left(1+\left|z_{\xi}\right|+\left|z_{g}\right|\right)
$$

\footnotetext{
${ }^{1}$ In the following, $\mathrm{O}(\zeta)$ denotes any function such that, for some constant $C,|\mathrm{O}(\zeta)(\varpi)| \leq C|\zeta(\varpi)|, \forall \varpi$.
} 
for some constant number $K$. Now, since (34) is always satisfied, $\left|z_{\xi}\right|$ is bounded along any solution of the controlled system so that:

$$
|\Delta| \leq \bar{K}\left(1+\left|z_{g}\right|\right)
$$

for some other constant number $\bar{K}$ (that depends on the initial conditions). It follows from (42) and the above inequality that, along any solution of the controlled system:

$$
\frac{d}{d t}\left|z_{g}\right|^{2} \leq \overline{\bar{K}}\left(\left|z_{g}\right|^{2}+1\right) .
$$

This inequality and the comparison lemma [32, Lem. 3.4] in turn imply that $\left|z_{g}\right|$ cannot grow faster than exponentially. Therefore, there is no finite-time explosion and $z_{g}(t)$ is well defined for any time $t \geq 0$. From (34), $z_{\xi}(t)$ is also well defined for any time $t \geq 0$, and so are $\alpha(t), \beta(t)$ since they belong to compact manifolds. This concludes the proof of existence of the controlled system solutions on $[0,+\infty)$.

We now derive ultimate bounds for $z_{g}$. Since $z_{\xi}(t)$ converges exponentially to zero as $t$ tends to infinity, one deduces from (44) that:

$$
\tilde{x}_{4,5,6}(t)-\left(h(\beta(t))+x_{4}^{\star}(\alpha(t), t) h_{3}(\beta(t)) e_{2}\right)
$$

converges to zero. The assumed boundedness of $\left|x_{4}^{\star}\right|$ and $|h|$ then yields the following ultimate bounds of $\left|\tilde{x}_{4}\right|$ and $\left|\tilde{x}_{5}\right|$ :

$$
\limsup _{t \rightarrow+\infty}\left|\tilde{x}_{4}(t)\right| \leq \varepsilon_{h}, \quad \limsup _{t \rightarrow+\infty}\left|\tilde{x}_{5}(t)\right| \leq \varepsilon_{h}\left(1+\varepsilon_{f}\right) .
$$

Recall from (47) that $\left|x_{5}^{\star}\right| \leq c\left(\left|z_{g}\right|+1\right)$ for some constant number $c>0$. Moreover, this number does not depend on $\varepsilon_{h}$ nor on the system's initial conditions. (43) then yields:

$$
|\Delta| \leq\left|\tilde{x}_{5}\right|\left(1+\varepsilon_{f}+\left|\tilde{x}_{4}\right|\right)+c\left|\tilde{x}_{4}\right|\left(\left|z_{g}\right|+1\right) .
$$

Furthermore, $\|\bar{R}\|=1$ and, from (17), $\left\|A_{g}\right\| \leq 1+\varepsilon_{f}$. In view of (42) one then deduces that:

$$
\begin{aligned}
\frac{d}{d t}\left|z_{g}\right|^{2} \leq & -2 z_{g}^{\top} K_{g} z_{g}+c\left(1+\varepsilon_{f}\right)\left|\tilde{x}_{4}\right|\left|z_{g}\right|^{2}+ \\
& \left(1+\varepsilon_{f}\right)\left[\left|\tilde{x}_{5}\right|\left(1+\varepsilon_{f}+\left|\tilde{x}_{4}\right|\right)+c\left|\tilde{x}_{4}\right|\right]\left|z_{g}\right| \\
\leq & -\left(2 \lambda_{\min }\left(K_{g}\right)-c\left(1+\varepsilon_{f}\right)\left|\tilde{x}_{4}\right|\right)\left|z_{g}\right|^{2}+ \\
& \left(1+\varepsilon_{f}\right)\left[\left|\tilde{x}_{5}\right|\left(1+\varepsilon_{f}+\left|\tilde{x}_{4}\right|\right)+c\left|\tilde{x}_{4}\right|\right]\left|z_{g}\right|
\end{aligned}
$$

with $\lambda_{\min }\left(K_{g}\right)>0$ denoting the smallest singular value of $K_{g}$. Let us now assume that:

$$
\varepsilon_{h}<\bar{\varepsilon}_{h}=: \frac{2 \lambda_{\min }\left(K_{g}\right)}{c\left(1+\varepsilon_{f}\right)} .
$$

Then, from (49), there exists $T>0$ such that, for $t \geq T$, $\left|\tilde{x}_{4}(t)\right|<\left(\varepsilon_{h}+\bar{\varepsilon}_{h}\right) / 2$. Thus, for $t \geq T$ :

$$
2 \lambda_{\min }\left(K_{g}\right)-c\left(1+\varepsilon_{f}\right)\left|\tilde{x}_{4}(t)\right|>c\left(1+\varepsilon_{f}\right)\left(\varepsilon_{h}+\bar{\varepsilon}_{h}\right) / 2>0 .
$$

This inequality together with (49) and (50) imply, by using the comparison lemma, that $\left|z_{g}\right|$ is ultimately bounded with:

$$
\begin{aligned}
\limsup _{t \rightarrow+\infty}\left|z_{g}(t)\right| & \leq \frac{\left(1+\varepsilon_{f}\right)\left[\varepsilon_{h}\left(1+\varepsilon_{f}\right)\left(1+\varepsilon_{f}+\varepsilon_{h}\right)+c \varepsilon_{h}\right]}{2 \lambda_{\min }\left(K_{g}\right)-c\left(1+\varepsilon_{f}\right) \varepsilon_{h}} \\
& \leq \frac{\varepsilon_{h}\left(1+\varepsilon_{f}\right)\left[\left(1+\varepsilon_{f}\right)\left(1+\varepsilon_{f}+\varepsilon_{h}\right)+c\right]}{2 \lambda_{\min }\left(K_{g}\right)-c\left(1+\varepsilon_{f}\right) \varepsilon_{h}} .
\end{aligned}
$$

This bound does not depend on the initial conditions and it tends to zero as $\varepsilon_{h}$ tends to zero. Since $|\tilde{g}| \leq\left|z_{g}\right|+\varepsilon_{f}$, $\tilde{g}$ is ultimately bounded by a value that tends to $\varepsilon_{f}$ as $\varepsilon_{h}$ tends to zero.

\section{REFERENCES}

[1] R. Brockett, "Asymptotic stability and feedback stabilization," in Differential Geometric Control Theory, R. M. R.W. Brockett and H. Sussmann, Eds. Birkauser, 1983.

[2] C. Samson, "Velocity and torque feedback control of a nonholonomic cart," Int. Workshop in Adaptative and Nonlinear Control: Issues in Robotics, 1990, also in LNCIS, Vol. 162, Springer Verlag, 1991.

[3] J.-M. Coron, "Global asymptotic stabilization for controllable systems without drift," Mathematics of Control, Signals, and Systems, vol. 5, pp. 295-312, 1992.

[4] A. Teel, R. Murray, and G. Walsh, "Nonholonomic control systems: from steering to stabilization with sinusoids," International Journal of Control, vol. 62, pp. 849-870, 1995, also in Proc. of the IEEE CDC, pp. 1603-1609, 1992.

[5] C. Samson, "Control of chained systems. application to path following and time-varying point-stabilization," IEEE Trans. on Automatic Control, vol. 40, pp. 64-77, 1995.

[6] O. J. Sørdalen and O. Egeland, "Exponential stabilization of nonholonomic chained systems," IEEE Trans. on Automatic Control, vol. 40, pp. 35-49, 1995.

[7] I. Kolmanovsky and N. McClamroch, "Developments in nonholonomic control problems," IEEE Control Systems, pp. 20-36, 1995.

[8] A. Astolfi, "Discontinuous control of nonholonomic systems," Systems \& Control Letters, vol. 27, pp. 37-45, 1996.

[9] R. M'Closkey and R. Murray, "Exponential stabilization of driftless nonlinear control systems using homogeneous feedback," IEEE Trans. on Automatic Control, vol. 42, pp. 614-628, 1997.

[10] P. Morin and C. Samson, "Practical stabilization of driftless systems on Lie groups: the transverse function approach," IEEE Trans. on Automatic Control, vol. 48, pp. 1496-1508, 2003.

[11] P. E. Crouch, "Spacecraft attitude control and stabilization: applications of geometric control theory to rigid body models," IEEE Trans. on Automatic Control, vol. 29, pp. 321-331, 1984.

[12] A. D. Lewis and R. M. Murray, "Configuration controllability of simple mechanical control systems," SIAM Journal on control and optimization, vol. 35, no. 3, pp. 766-790, 1997.

[13] P. Morin, C. Samson, J.-B. Pomet, and Z.-P. Jiang, "Time-varying feedback stabilization of the attitude of a rigid spacecraft with two controls," Systems \& Control Letters, vol. 25, pp. 375-385, 1995.

[14] J.-M. Coron and E.-Y. Kerai, "Explicit feedbacks stabilizing the attitude of a rigid spacecraft with two control torques," Automatica, vol. 32, pp. 669-677, 1996.

[15] P. Morin and C. Samson, "Control with transverse functions and a single generator of underactuated mechanical systems," in IEEE Conf. on Decision and Control, 2006, pp. 6110-6115.

[16] B. d'Andréa Novel and S. Thorel, "Control of non holonomic or underactuated mechanical systems: The examples of the unicycle robot and the slider," ESAIM: Control, Optimisation and Calculus of Variations, vol. 22, no. 4, pp. 983-1016, 2016.

[17] J. Ostrowski, J. Burdick, A. D. Lewis, and R. M. Murray, "The mechanics of undulatory locomotion: The mixed kinematic and dynamic case," in Robotics and Automation, 1995. Proceedings., 1995 IEEE International Conference on, vol. 2. IEEE, 1995, pp. 1945-1951.

[18] J. Ostrowski, A. Lewis, R. Murray, and J. Burdick, "Nonholonomic mechanics and locomotion: the snakeboard example," in Robotics and Automation, 1994. Proceedings., 1994 IEEE International Conference on. IEEE, 1994, pp. 2391-2397.

[19] J. P. Ostrowski and J. W. Burdick, "Controllability tests for mechanical systems with symmetries and constraints," J. Appl. Math. Comp. Sci, vol. 7, no. 2, pp. 101-127, 1997.

[20] J. Ostrowski and J. Burdick, "The geometric mechanics of undulatory robotic locomotion,", The International Journal of Robotics Research, vol. 17 , no. 7, pp. 683-701, 1998.

[21] A. Kuleshov, "Further development of the mathematical model of a snakeboard," Regular and Chaotic Dynamics, vol. 12, no. 3, pp. 321334, 2007.

[22] F. Bullo and A. D. Lewis, "Kinematic controllability and motion planning for the snakeboard," IEEE Transactions on Robotics and Automation, vol. 19, no. 3, pp. 494-498, 2003.

[23] E. A. Shammas, H. Choset, and A. A. Rizzi, "Towards a unified approach to motion planning for dynamic underactuated mechanical systems with non-holonomic constraints," The International Journal of Robotics Research, vol. 26, no. 10, pp. 1075-1124, 2007.

[24] E. Shammas and M. De Oliveira, "Motion planning for the snakeboard," The International Journal of Robotics Research, vol. 31, no. 7, pp. 872885, 2012. 
[25] B. Varszegi, D. Takacs, G. Stepan, and S. J. Hogan, "Stabilizing skateboard speed-wobble with reflex delay," Journal of The Royal Society Interface, vol. 13, no. 121, 2016.

[26] P. Morin and C. Samson, "Control of nonholonomic mobile robots based on the transverse function approach," IEEE Trans. on Robotics, vol. 25, pp. 1058-1073, 2009.

[27] M. Ishikawa, "Trident snake robot: locomotion analysis and control," in IFAC Symp. on Nonlinear Control Systems, 2004, pp. 1169-1174.

[28] M. Ishikawa, P. Morin, and C. Samson, "Tracking control of the trident snake robot with the transverse function approach," in IEEE Conf. on Decision and Control.

[29] M. Fliess, J. Lévine, P. Martin, and P. Rouchon, "Flatness and defect of non-linear systems: introductory theory and examples," International journal of control, vol. 61, no. 6, pp. 1327-1361, 1995.

[30] A. Lewis, J. Ostrowski, J. Burdick, and R. Murray, "Nonholonomic mechanics and locomotion; the snakeboard example," in Proceedings of the IEEE International Conference on Robotics and Automation (ICRA), 1994, pp. 2391-2400.

[31] J. Ostrowski, J. Burdick, A. Lewis, and R. Murray, "The mechanics of undulatory locomotion: the mixed kinematic and dynamic case," in Proceedings of the IEEE International Conference on Robotics and Automation (ICRA), 1995, pp. 1945-1951.

[32] H. Khalil, Nonlinear systems, 3rd ed. Prentice Hall, 2002.

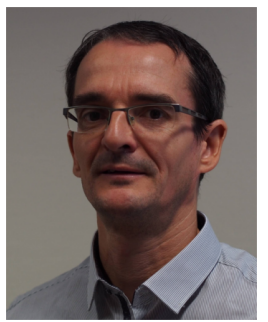

Pascal Morin received the Maitrise degree from Université Paris-Dauphine in 1990, and the Diplôme d'Ingénieur and $\mathrm{PhD}$ from Ecole des Mines de Paris in 1992 and 1996 respectively. He spent one year as a post-doctoral fellow in the Control and Dynamical Systems Department at the California Institute of Technology. He was Chargé de Recherche at INRIA, France, from 1997 to 2011 . From 2011 to 2016 he was in charge of the "Chaire RTE-UPMC Minidrones autonomes" at ISIR, a Laboratory of Université Pierre et Marie Curie (UPMC) and CNRS He is now Professor at Sorbonne Université. His research interests include stabilization and estimation problems for nonlinear systems, and applications to mechanical systems such as nonholonomic vehicles or UAVs.

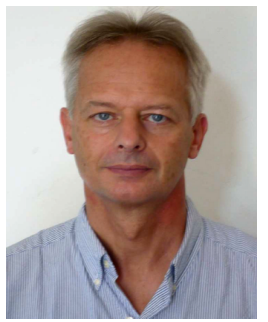

Claude Samson graduated from the Ecole Supérieure d'Electricité in 1977, and received Docteur-Ingénieur $(\mathrm{PhD})$ and Docteur d'Etat degrees from the Mathematics and Computer Science University of Rennes, France, in 1980 and 1983 respectively, both in the domain of Physical Sciences with a speciality in Automatic Control. He joined INRIA, the French institute in Computer Science and Automatic Control, in 1981, where he is currently Directeur de Recherche. In 2014 he joined the I3S Laboratory in Nice/Sophia-Antipolis to conduct collaborative research on modeling, state-estimation, and control of mechanical systems with applications to autonomous vehicles. Dr. Samson is the coauthor, with M. Leborgne and B. Espiau, of the book Robot Control The Task-Function Approach (Oxford University Press, 1991). With Prof Pascal Morin (ISIR-University of Pierre and Marie Curie, Paris) he initiated the development of the Transverse Function control approach for critical nonlinear systems (http://www.transversefunction.com/). 